\title{
Salesperson Market Orientation Behavior: Its Antecedents and the Mediating Role of Working Smart Behaviors in Sales Performance
}

\author{
Yen-Chun Chen, Adriana Amaya Rivas, and Wann-Yih Wu
}

\begin{abstract}
Recent research highlights the importance of market orientation in service providers. In the current knowledge-intensive economy, salespeople act more as a knowledge brokering role by transferring knowledge to customers instead of only convincing customers to buy products so as to create superior customer value. Therefore, this paper proposes a research model to identify the antecedents of salesperson market orientation behavior (SMOB) as well as its impacts on working smart behaviors (i.e., sales planning and adaptive selling) and sales performance. More specifically, the research model comprises three sets of antecedents of SMOB: salesperson goal orientation; sales control systems and sales management support. The research model and hypotheses are tested using data obtained from a sample of 264 Taiwanese salespeople in the financial service industry. The empirical findings suggest that salesperson learning orientation and behavioral controls are significantly and positively related to SMOB. The results also show that SMOB has positive impacts on both sales planning and adaptive selling, which in turn achieve better sales performance. These results provide some new insights into the sales management literature.
\end{abstract}

Keywords Salesperson market orientation behavior $\bullet$ Salesperson goal orientation

- Sales control systems • Sales planning • Adaptive selling

\section{References available on request.}

Y.-C. Chen $(\bowtie) \bullet$ A.A. Rivas

Chinese Culture University, Taipei City, Taiwan

e-mail: cyj22@ulive.pccu.edu.tw; adrianaamayarivas@gmail.com

W.-Y. Wu

Nanhau University, Dalin Township, Taiwan

e-mail:wanyi@nhu.edu.tw 